\title{
Building a Culture of Critical and Creative Thinking. Creating and Sustaining Higher-Order Thinking as part of a Quality Enhancement Plan
}

\author{
Tania Allen', Sara Queen², Maria Gallardo-Williams³ ${ }^{3}$ Lisa Parks ${ }^{4}$, Anne Auten ${ }^{5}$, Susan \\ Carson $^{6}$ \\ ${ }^{1}$ Art \& Design, NC State University, US, ${ }^{2}$ Architecture, NC State University, US, \\ ${ }^{3}$ Chemistry, NC State University, US, ${ }^{4}$ Biological Sciences, NC State University, US, \\ ${ }^{5}$ University Honors, NC State University, US, ${ }^{6}$ Plant \& Microbial Biology, NC State \\ University, US.
}

\begin{abstract}
The TH!NK initiative at North Carolina State University seeks to bridge the gap between evidence-based research on teaching and actual teaching practices in the classroom. Through this work, the culture of teaching and learning on our campus is being transformed from teacher-centered to studentcentered instruction that promotes higher-order thinking across a diverse array of disciplines. Participating faculty engage in intensive faculty development; create discipline-specific classroom activities and assignments; become adept at providing students feedback on their thinking skills; and engage in a learning community to share and provide peer feedback on pedagogical innovations. The primary student learning outcome (SLO) is for students to apply critical and creative thinking skills and behaviors in the process of solving problems and addressing questions. Methods to achieve the institutional transformation include implementation of comprehensive faculty development focused on the use of evidence-based pedagogy that promotes higher-order thinking and rigorous outcomes assessment to provide means for continual improvement. The program has expanded into multiple phases, and involves strategies to create a more sustainable culture of critical and creative thinking through formal and informal learning and scholarship.
\end{abstract}

Keywords: higher-order thinking; quality enhancement; critical and creative thinking; program development; teaching strategies. 


\section{Background to the Quality Enhancement Program}

At North Carolina State University, we have seen increasingly higher standardized test scores and grade point averages in our incoming students. At the same time, our alumni report that they were not fully prepared for career challenges related to critical and creative thinking. This gap was the driving force behind NC State’s 2014 Quality Enhancement Plan, focused on enhancing higher-order thinking skills, including both critical and creative thinking, as a core part of both formal and informal learning throughout the University.

Robert Ennis (1995) defines critical thinking as reflective and reasonable thinking that is focused on deciding what to believe or do. He argues that critical thinking is a practical activity that includes creative actions such as raising questions, formulating hypotheses, generating alternatives, and gathering information. Popular frameworks that are effective in describing the evaluative nature of higher-order thinking often do not touch deeply on the creative aspects (Paul and Elder, 2012). Roger Martin, Dean of the Rotman School of Management at the University of Toronto, argues that critical and creative thinking are critical to the future of work and economic prosperity. He describes a long-term statistical trend away from routine-oriented work, which emphasizes rote learning and repetition, and toward more creativity-oriented jobs that emphasize critical analysis and making judgments (Martin, 2009, p.5). A 2012 IBM study of over 1,700 CEOs also reflects the changing nature of industry when reporting that the top 4 traits desired in the contemporary workforce were collaboration, communication, creativity and flexibility (Leading Through Connections, 2012, p.21).

\section{The TH!NK Program}

The NC State Quality Enhancement Plan, TH!NK, initially focused on training faculty teaching courses that serve primarily first-semester freshmen. It has since expanded to include faculty teaching courses at all academic levels, vertical integration through majors, faculty scholarship programs, and a teaching strategy database and resource sheets. The faculty training program seeks to bridge the gap between evidence-based research on teaching and teaching practices in the classroom. Through this work, we aim to transform the culture of teaching away from teacher-centered instruction and toward student-centered instruction that promotes higher-order thinking.

Participating faculty engage in intensive faculty development and create discipline-specific activities and assignments that utilize evidence-based pedagogical strategies that impact student higher-order thinking. They become adept at providing students feedback on their thinking skills, rather than just their final work products. They engage in a learning community where faculty share and provide peer feedback on pedagogical innovations and are supported in publicly disseminating pedagogical innovations. 
The primary student learning outcome we aim to achieve through the initiative is for students to apply critical and creative thinking skills and behaviors in the process of solving problems and addressing questions. Figure 1 illustrates the creative process, encompassing criticality and self-reflection, as we have defined it. Defining these skills explicitly not only helps students, but helps faculty mentors focus on building scholarship in the students. This process aligns directly with our student learning outcomes and our methods of assessment.

The key skills and behaviors we focus on are raising questions and formulating problems; gathering and assessing relevant information, synthesizing and generating ideas, considering alternatives, reaching reasoned conclusions, effectively communicating and reflecting at each stage of the process.

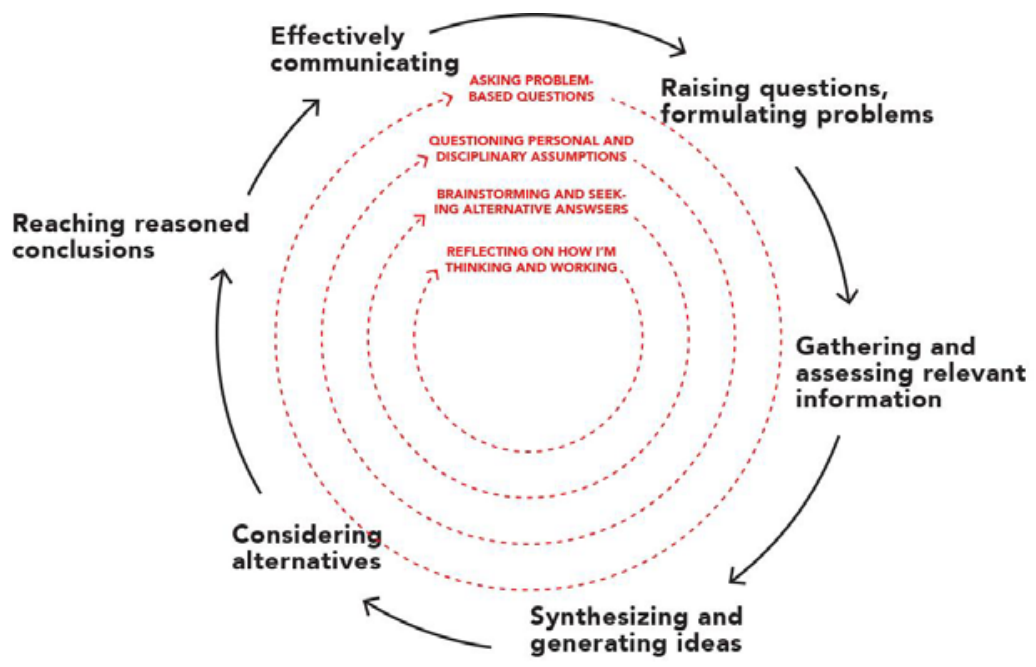

Figure 1. The critical and creative process.

\subsection{Program Structure}

The TH!NK initiative utilizes a peer mentoring model. The implementation team consists of a Director who is a faculty member in a STEM discipline and was engaged in the scholarship of teaching and learning prior to her directorship, four rotating TH!NK Fellows who are active faculty members and receive one course buy-out and \$5,000 additional compensation annually, half-time administrative support, as well as support from the Office of Assessment and the Office of Faculty Development. The Director and Fellows are responsible for workshop and resource development, as well as peer mentoring support as participants work on their course enhancements. The Office of Assessment is responsible for student learning outcomes assessment, and the Office of Faculty Development assesses our workshops and faculty learning. 


\subsection{Faculty Development Plan}

Approximately thirty faculty members are recruited to the TH!NK Faculty for training and participation each year. The intensive TH!NK Institute takes place in May, the week following commencement. TH!NK Faculty work on course revisions over the summer with the support of their interdisciplinary peer cluster consisting of other TH!NK Faculty and a TH!NK Fellow, and we reconvene in August prior to the start of the semester for faculty to share out their pedagogical innovations and to receive structured peer feedback.

The primary goal of the May institute is to define the critical and creative thinking process across disciplines and provide faculty with tools to create opportunities for students to practice the skills in the process, as well as tools to provide feedback on students' thinking process in addition to their work product. Topics that faculty engage with in the workshops include: stages of epistemological growth (Perry, 1981; Paulesen and Feldman, 1999; Wood and Kardash, 2002); the critical and creative thinking process (Ennis, 1985; Sternberg and Lubart, 1999; Csikszentmihalyi, 1996); introducing the intellectual standards of critical and creative thinking (Paul and Elder, 2012); peer evaluation (Nystrand,1989; Volz, 2009); assessing creative thinking in the classroom (AAC\&U Value Rubrics; Stein et al, 2006); divergent and convergent thinking (Schommer, 1990); critical thinking scenarios (Carson, 2015a); data visualization and concept mapping (Novak, 1990); writing to think (Anson and Beach, 1995; Angelo and Cross, 1993); and metacognition and self-reflection (Paulesen and Feldman, 1999; Bruning et al, 1995; Hofer and Pintrich, 1997; Schommer, 1990).

\subsection{Faculty Participation}

Over 130 faculty, representing all undergraduate-serving colleges at NC State, have participated as TH!NK Faculty over the past five years. Faculty survey responses indicated that $>95 \%$ of TH!NK Faculty made enhancements not only to their TH!NK course, but to other courses they teach. In addition, many TH!NK Faculty and Fellows have published or presented scholarship related to their TH!NK work. References to these papers (8) and proceedings (18) are available at https://think.dasa.ncsu.edu/.

It is important to emphasize that while the TH!NK Faculty were "trainees," they made tremendous contributions to every aspect of the faculty development over the past 5 years. Many contributed constructive feedback on our training and resources, provided examples of exceptional and innovative discipline-specific classroom activities and assignments, and all provided peer support and feedback to other participants.

\section{TH!NK Faculty Scholarship Development}

We have formalized a research track for TH!NK Faculty alumni (TH!NK Researchers Program) to provide training on how to collect data and publish research projects based on 
their redesigned courses. These studies measure changes in student learning as a result of the innovations introduced by the TH!NK program. A series of workshops for the TH!NK Faculty gives a framework for beginning their SoTL research. These workshops provide the structure for continued participation in the cohort, individual mentorship, and financial support to attend disciplinary conferences. Each participant receives \$2000 to travel to present work at a conference or to use toward professional development activities.

This structural and financial support helps new and emerging SoTL researchers and faculty with prior publishing experience to form a learning community. In many departments, faculty with a primary teaching role lack a mechanism for engaging in SoTL research and publishing, yet it is becoming increasingly more important in the tenure, promotion, and review process. Training included support in the research process, and covered the following areas: introduction to the scholarship of teaching and learning; steps of the research process/ basic study design; generating research ideas: new idea vs. using existing data; outlets for presentation/publication; qualitative methods; the institutional review board (irb) process; educational data collection and analysis; writing support; presentation and dissemination of results; challenges when doing educational research; and grant applications for further funding.

Preliminary outcomes of the first cohort indicate that the faculty involved have been actively submitting conference abstracts and preparing journal articles. Program participants have reported increased confidence in their ability to share their educational findings as a result of their participation in the TH!NK Researchers Program.

\section{Sustaining the Program}

To sustain the impact of TH!NK across campus, a culture of self-sufficiency was a primary focus for the second phase of the TH!NK program. In addition to the TH!NK Researchers Program mentioned above, we have developed multiple stand-alone resources for faculty.

In Spring 2018, TH!NK Fellows compiled teaching and SoTL research resources on the TH!NK website. These resources serve two main functions: 1) to reinforce faculty training for TH!NK alumni and 2) to provide additional faculty TH!NK program resources. All handouts, slide presentations, and assessment tools presented in the May and August teacher training as well as the SoTL researcher training are accessible. Additionally, the website contains evidence-based Teaching Strategy Resources Sheets which provide overviews of a variety of teaching strategies, offer 3-4 examples of college level exercises which use the strategy in different disciplinary or class-type settings, and provide sources for further information. Each of these Teaching Strategy Resource Sheets describes how the featured strategy fits within the critical and creative process. Another key component to the online resources is an Assignment Database which is populated with original classroom assignments 
and activities created by NC State THINK Faculty. Each entry is tagged by discipline, the scale of the activity or assignment, and most importantly, the skills in the critical and creative thinking process that students must employ. The database is accessible at https://apps.dasa.ncsu.edu/think/public/index.php.

\section{Preliminary Outcomes and Assessment}

We are in the process of gathering summative data on the student learning outcomes of the first five years of the initiative. Preliminary assessment is discussed below.

Over 11,000 students (enrollments) have been impacted directly in TH!NK sections to date. However, we know that the number of students impacted is much greater, with $95 \%$ of TH!NK Faculty reporting enhancements to their other sections and classes.

We use three measures of assessment for student learning outcomes: the Critical-thinking Assessment Test (CAT) developed by Tennessee Tech; the TH!NK Common Rubric that is based on AAC\&U VALUE rubric and current research on creativity; and student surveys. A bird's eye view of the data points to gains in a greater number of different critical thinking skills as assessed on the CAT in 2015 and 2016 compared to the 2013 pre-TH!NK baseline year. Prior to implementation, planners of the QEP targeted the "emerging” level on the Common Rubric as the level we would hope to bring our students to over the course of their first semester. On average, we hit that benchmark for most rubric dimensions. The dimensions of "intellectual risk taking" and "recognizing assumptions" appear to be the areas where our students struggle the most. Regardless, our students have plenty of room to grow in the skills identified in the Common Rubric, and that is one reason why we decided to expand TH!NK into upper-level courses.

We use multiple measures of student growth and ability because each instrument has strengths and limitations. The CAT measures a number of transferable critical thinking skills in a discipline non-specific manner. This allows us to use this as a common instrument across classes/majors, and because the scoring is standardized, it reduces scorer subjectivity. The limitations of the CAT include the exclusion of many of the creative thinking skills we want to impact; our incoming freshmen score very high on this test, and therefore we see a ceiling effect in some items for a large portion of our students; and we do face concerns over effort during test taking for more advanced students compared to incoming freshmen since there is not a grade motivator.

The TH!NK Common Rubric assesses the critical and creative thinking skills that NC State University's QEP identified as outcomes it wanted our students to achieve since it was tailored specifically to our program. Unlike the CAT, this rubric is applied to work that students did as a part of their course, and so most are motivated to try their best because the 
assignment contributes to their class grade. Limitations of this rubric is that every assignment is different. Some assignments may have more room for critical and creative thinking than others, and although we hold required rubric norming sessions, scoring does have some subjectivity.

\section{Conclusion}

In continuing to grow and expand the TH!NK program, we continue to look for new opportunities to encourage a culture of critical and creative thinking that includes more and more informal learning opportunities. Now in its 5th year, the TH!NK program has been successful in creating a culture where faculty engage more intentionally in integrative learning in order to help students go deeper into their inquiry and reflection. But there is still room to grow in providing consistent opportunities in a coordinated manner across all curricula, to guide students in threading their varied experiences together in a significant way, and to initiate opportunities-inside and outside of the classroom-to seek out deeper knowledge around areas of interest that support student inquiry in new and innovative ways. There are also opportunities to leverage resources that are currently available (e.g. General Education requirements) to make those courses and experiences aligned with the higher order thinking skills that are at the core of the program.

\section{References}

Angelo, T.A. and Cross, K.P. (1993). Classroom Assessment Technologies (Second Edition). San Francisco: Jossey-Bass Publishers.

Anson, C. and R. Beach. 1995. Journals in the Classroom: Writing to Learn. Norwood, MA. Christopher Gordon Publishers.

Bruning, R., G. Schraw, R. Ronning. 1995. Cognitive Psychology and Instruction. Upper Saddle River, NJ: Prentice Hall.

Carson, S. 2015a. Targeting critical thinking skills in a first-year undergraduate research course. JMBE. 16 (2): 148-156.

Csikszentmihalyi, M. 1996. Creativity: Flow and the Psychology of Discovery and Invention. New York, NY. Harper Collins Publishers.

Ennis, R.H. (1995). Critical Thinking. New Jersey: Pearson FT Press. ISBN-13: 9780133747119

Hofer, B. and P. Pintrich. 1997. The development of epistemological theories: Beliefs about knowledge and knowing and their relation to learning. Review of Educational Research. 67: 88-140.

Leading Through Connections. Insights from the Global Chief Executive Officer Study. (2012). New York: IBM Global Business Services. GBE03485-USEN-01

Martin, R. (2009). The Design of Business: Why Design Thinking is the Next Competitive Advantage. Cambridge: Harvard Business Review Press. ISBN-10: 9781422177808. 
Novak, J. D. 1990. Concept mapping: A useful tool for science education. Journal of Research Science Teaching. 27:937- 949.

Nystrand, M. and D. Brandt. 1989. Response to writing as a context for learning to write. In Anson, C.,Writing and response: Theory, practice, and research: Urbana, IL: National Council of Teachers of English. p. 209-230.

Paul, R and Elder, L. (2012). Critical Thinking : Tools for Taking Charge of Your Learning and Your Life. New Jersey: Pearson FT Press. ISBN: 9780133115284.

Paulesen, M. and K. Feldman. 2002. Student motivation and epistemological beliefs. New Directions for Teaching and Learning. Summer 1999. 78. 17-25.

Perry, W. G. 1981. Cognitive and ethical growth: The making of meaning. In A. W. Chickering \& Assoc. (Eds.), The modern American college (pp. 76-116). San Francisco: Jossey-Bass.].

Schommer, M. (1990). Effects of beliefs about the nature of knowledge on comprehension. Journal of Educational Psychology. 82 (3). 498-504.

Stein, B., A. Haynes, and M. Redding. 2006. Project CAT: Assessing Critical Thinking Skills. Proceedings of the National STEM Assessment Conference. NSF and Drury University.

Sternberg, R. and T. Lubart. 1999. Concepts of creativity: Prospects and paradigms. Handbook of Creativity. New York, NY: Cambridge University Press.

Volz, T., and A. Saterbak. 2009. Students' strengths and weaknesses in evaluating technical arguments as revealed through implementing Calibrated Peer Review ${ }^{\mathrm{TM}}$ in a bioengineering laboratory. [Special issue on Writing Technologies and Writing Across the Curriculum] Across the Disciplines, 6. Retrieved December 11, 2015, from http://wac.colostate.edu/atd/technologies/volz_saterbak.cfm

Wood, P. and C. Kardash. 2002. Critical elements in the design and analysis of studies of epistemology. In B. K. Hofer, P. Pintrich (eds.). Personal epistemology: The psychology of beliefs about knowledge and knowing. Mahwah, NJ: Erlbaum. 231-260.

\section{Assessment References}

Cat Test: https://www.tntech.edu/cat/

AAC\&U Value Rubrics: https://www.aacu.org/value-rubrics

TH!NK Common Rubric: https://think.dasa.ncsu.edu/wp-content/uploads/sites/

20/2018/05/11-Common-Rubric-2018.docx.pdf

THINK student survey: https://think.dasa.ncsu.edu/reflection-survey/. 